\title{
Use of Acyl-Homoserine Lactones Leads to Improved Growth of Ginseng Seedlings and Shifts in Soil Microbiome Structure
}

\author{
Jerald-Conrad Ibal ${ }^{1, \dagger}$, Min-Kyu Park ${ }^{1, \dagger}{ }^{+}$Gun-Seok Park ${ }^{2}$, Byung-Kwon Jung ${ }^{3}$, Tae-Hyung Park ${ }^{4}$, \\ Min-Sueng Kim ${ }^{4}$, Gi-Ung Kang ${ }^{5}$, Yeong-Jun Park ${ }^{1}$ and Jae-Ho Shin ${ }^{1,4, *(D)}$ \\ 1 Department of Applied Biosciences, Kyungpook National University, Daehak-ro 80, Daegu 41566, Korea; \\ jerald.ibal@gmail.com (J.-C.I.); pmk601313@knu.ac.kr (M.-K.P.); yjpark1091@knu.ac.kr (Y.-J.P.) \\ 2 Atogen Co., Ltd., Yuseong-gu, Daejeon 34015, Korea; gspark@atogen.co.kr \\ 3 CJ Blossom Park, Yeongtong-gu, Suwon-si 16495, Korea; bk.jung@cj.net \\ 4 Department of Integrative Biotechnology, Kyungpook National University, Daehak-ro 80, \\ Daegu 41566, Korea; taehyung.park@knu.ac.kr (T.-H.P.); chahun4270@knu.ac.kr (M.-S.K.) \\ 5 Deutsches Rheuma-Forschungszentrum (DRFZ), Institute of the Leibniz Association, 10117 Berlin, Germany; \\ kanggiung5748@gmail.com \\ * Correspondence: jhshin@knu.ac.kr; Tel.: +82-53-950-5716 \\ + These authors are contributed equally.
}

\section{check for} updates

Citation: Ibal, J.-C.; Park, M.-K.; Park, G.-S.; Jung, B.-K.; Park, T.-H.; Kim, M.-S.; Kang, G.-U.; Park, Y.-J.; Shin, J.-H. Use of Acyl-Homoserine Lactones Leads to Improved Growth of Ginseng Seedlings and Shifts in Soil Microbiome Structure. Agronomy 2021, 11, 2177. https://doi.org/ 10.3390/agronomy11112177

Academic Editors: Blanca R. López and Luz E. de-Bashan

Received: 26 September 2021

Accepted: 26 October 2021

Published: 28 October 2021

Publisher's Note: MDPI stays neutral with regard to jurisdictional claims in published maps and institutional affiliations.

Copyright: (c) 2021 by the authors. Licensee MDPI, Basel, Switzerland. This article is an open access article distributed under the terms and conditions of the Creative Commons Attribution (CC BY) license (https:/ / creativecommons.org/licenses/by/ $4.0 /)$.

\begin{abstract}
Panax ginseng is a well-known medicinal plant that achieves strong resistance against plant pathogens while growing in the wild. Due to the high market demand for ginseng as a health food source, ginseng cultivation is prevalent in South Korea. However, continuous monocropping creates problems like irregular growth or vulnerability to crop diseases. Quorum sensing (QS) deals with the intracellular communication of bacteria and plays a role in dynamic changes in the soil microbiome. Here, we investigated how acyl-homoserine lactone (AHL) signaling molecules in QS (C8, C10, and C12) improve plant growth and induce shifts in the soil microbiome. To assess the effects, we recorded root and shoot growth of ginseng seedlings and checked the changes in the soil microbiome during different time points $(0,2,4$, and 8$)$ after 8 weeks of growth. We observed that soils treated with $\mathrm{N}$-decanoyl- ${ }_{\mathrm{L}}$-homoserine lactone (C10) showed the most pronounced effects. Very striking was that $\mathrm{C} 10$ had the lowest alpha diversity. Using Phylogenetic Investigation of Communities by Reconstruction of Unobserved States (PICRUSt2), we observed a high number of QS-related functional genes, with the highest count occurring in the untreated planted soil (W). Together with the known direct and beneficial effects of AHLs on plant development, AHLs treated mono-cropped soil showed trends in the microbiome community.
\end{abstract}

Keywords: ginseng; soil microbiome; quorum sensing

\section{Introduction}

Panax ginseng C. A. Meyer, also known as Korean or Asian ginseng, from the Araliaceae family, has been used as a traditional herbal medicine for thousands of years in East Asia. Primarily grown in Korea, China, Japan, and Russia, P. ginseng is considered an essential crop due to the ginseng saponins it produces, which have pharmacological properties $[1,2]$. It is regarded as one of the most extensively used herbal medicines and is reported to have a wide range of applications in therapeutic and pharmacological industries [3]. Since it is a herbaceous perennial plant, it takes at least 5-6 years for ginseng to reach marketable size, while some strains cultivated in mountain forests take longer than 10 years to mature [4,5]. Due to the long cultivation time, the rhizosphere microbial community plays a vital role in ginseng growth, contributing via nutrient solubilizing, plant growth promotion, and protection against disease [6,7].

However, a big problem in the cultivation of ginseng is continuous monocropping. Problems linked to continuous monocropping occur in various crop species, including 
apple, cherry, alfalfa, rice, corn, and strawberry. These problems are usually related to the deterioration of soil physicochemical properties, allelopathy/autotoxicity, soil-borne diseases, and changes in the soil microbial communities [8]. A previous study on the continuous cropping of Asian ginseng (Panax ginseng) proposed that changes in the rhizosphere microbiome after the addition of inorganic fertilizers are a key factor resulting in the replant problem [9].

In a complex environment, plants interact with specific soil microorganisms that inhabit the area around the root, known as the rhizosphere [10]. One of the most intricate ecosystems on earth, the rhizosphere serves as a hotspot for millions of microbial cells [11]. The interactions of microorganisms that coexist in the rhizosphere are active, and changes in metabolites exuded by plant roots help shape the composition of the root microbiota [12]. This complex web of interactions may directly or indirectly affect plant growth. Since bacteria are the most abundant microorganisms in the rhizosphere and are highly competitive in root colonization, they probably influence plant physiology to a greater extent than other microorganisms [13]. As such, root-microbe interaction is essential for plants, offering protection from diseases and tolerating abiotic stresses, among other benefits for plant health [14].

Quorum sensing (QS) is a process through which bacteria attain intercellular communication. The production and detection of autoinducer signaling molecules make it possible for bacteria to observe cell density and coordinate behavioral changes collectively [15]. Signaling molecules allow for gene regulation to occur within the population, allowing the bacterial community to regulate cellular processes-such as biofilm formation, motility, sporulation, and virulence-which are important for survival and adaptation in the environment [16]. Microbially derived signal molecules are assigned into one of two main categories: (i) amino acids and short peptide pheromones commonly utilized by Gram-positive bacteria $[17,18]$ and (ii) fatty acid derivatives such as $N$-acyl homoserine lactones (AHLs) that are utilized by Gram-negative bacteria. Gram-positive bacteria use autoinducing peptides (AIP) as signaling molecules. In contrast, the most common autoinducers in gram-negative bacteria are AHLs, containing a primary $\mathrm{N}$-acylated homoserine lactone ring and $4-18$ carbon acyl chains, with variations $[16,19]$.

A number of studies have shown that plant responses to certain AHL molecules are specific depending on the length of the acyl moiety [20-22]. Not only do AHL molecules affect plant responses, but a study by Liang et al. [23] showed that a variety of AHLs can affect the bacterial community. In this study, we hypothesize that treatment of AHL could be potentially used to alleviate the problems that occur during monocropping by shifting the soil microbiome in addition to their plant growth promoting activity. We investigated the effect of using three AHL signaling molecules on the mono-cropped ginseng soil microbiome. Moreover, we examined whether different moieties of AHL could alleviate soil sickness caused by continuous monocropping through the microbial community shift and observation of the phenotype and survival incidence of ginseng soil seedlings during a two-month growth period. To address these, we took soil samples from different time points $(0,2,4,6$, and 8 weeks) of ginseng seedlings planted in pots (with and without AHL treatment) and checked the microbiome through $16 \mathrm{~S}$ rRNA amplicon sequencing. Using Phylogenetic Investigation of Communities by Reconstruction of Unobserved States (PICRUSt 2), we examined the functional genes present in the microbiome, specifically those involved in QS such as LuxI and LuxR genes, then determined the correlating growth of seedlings by observing their growth parameters.

\section{Materials and Methods}

\subsection{Ginseng Soil Sampling}

Continuously cropped ginseng cultivation sites were located in Punggi $\left(36^{\circ} 48^{\prime} 37.6^{\prime \prime}\right.$ $\left.\mathrm{N} 128^{\circ} 32^{\prime} 28.5^{\prime \prime} \mathrm{E}\right)$, South Korea. Three soil cores (10 cm in depth rhizosphere soil) were randomly collected after removing ginseng plants and surface coverings for each field. The collected samples from the cores were then mixed. A total of $75 \mathrm{~kg}$ of soil samples were 
collected. Subsequently, soil samples considered 'unhealthy' (soils that grow unhealthy ginseng) were collected and used as a control.

\subsection{In Vitro Pot Experiment and Treatment of Quorum-Sensing Signaling Molecules in Ginseng}

A total of $75 \mathrm{~kg}$ of soil was placed in sterile plastic bags and sifted. After sifting, the soils were transferred into styrofoam pots containing $5 \mathrm{~kg}$ of soil. Three species of $\mathrm{N}$-AHL $\left(10 \mu \mathrm{M}\right.$ in $100 \mathrm{~mL}$ of $\mathrm{dH}_{2} \mathrm{O}$ of $\mathrm{N}$-octanoyl-L-homoserine lactone (C8), $\mathrm{N}$-decanoyl-

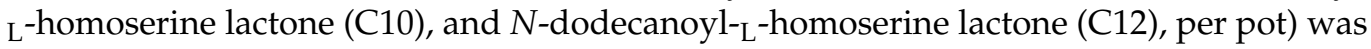
added. A control group of pots, which was only treated with water, was prepared monocropped soil (W) and non-planted bulk soil (BS) from the same sampling site. Four pieces of the 2-year-old ginseng seedlings were then planted in each pot. Each treatment was performed using three replicates, and the pots were placed in a growth chamber with an average temperature of $22{ }^{\circ} \mathrm{C}$ and average humidity of $60 \%$ for 2 months. Irrigation was performed every 7 days to ensure constant soil moisture. Around $5 \mathrm{~g}$ of soil sample was collected at different time points after planting ( 0 weeks, 2 weeks, 4 weeks, and 8 weeks) for 2 months and were stored at $-70^{\circ} \mathrm{C}$ for subsequent analysis. Growth parameters such as root and shoot length and the wet and dry biomass were recorded.

\subsection{DNA Extraction, PCR Amplification, and Sequencing}

Extraction of $0.5 \mathrm{~g}$ soil genomic DNA using the DNeasy PowerSoil Kit (Qiagen, Valencia, CA, USA) was performed following the manufacturer's instructions. The quality of the extracted DNA was assessed through electrophoresis and quantified using Qubit fluorometer 2.0 (Waltham, MA, USA). The extracted DNA was used to amplify the V4-V5 regions of the prokaryotic $16 \mathrm{~S}$ rRNA genes by polymerase chain reaction (PCR) with an initial denaturation at $95^{\circ} \mathrm{C}$ for $5 \mathrm{~min}$, followed by five cycles of annealing at $57^{\circ} \mathrm{C}$ for $30 \mathrm{~s}$ and extension at $72{ }^{\circ} \mathrm{C}$. The initial five cycles were succeeded by another 25 cycles at $95{ }^{\circ} \mathrm{C}$ for denaturation and $72{ }^{\circ} \mathrm{C}$ for annealing and extension. For PCR, we used the universal primers 515 $\mathrm{F} 5^{\prime}$-barcode-GTGCCAGCMGCCGCGGTAA- $3^{\prime}$ and $907 \mathrm{R} 5^{\prime}$ barcode-CCGYCAATTCMTTTRAGTTT- $3^{\prime}$. The barcode is an eight-base sequence unique to each sample so that each read can be identified and sorted before data analysis. An Agilent 2100 Bioanalyzer (Santa Clara, CA, USA) was used to check for the correct concentration needed for the sequencing platform. The amplified regions were then pooled and sequenced using the Illumina MiSeq platform (San Diego, CA, USA), following the manufacturer's protocols.

\subsection{Bioinformatic Analysis}

The raw FASTQ files were processed using the next-generation microbiome bioinformatics platform Quantitative Insights into Microbial Ecology (QIIME) 2 [24]. Quality filtered reads were then input into the QIIME 2 plugin Deblur [25] to produce amplicon sequence variants (ASV). A trim length of 250 base pairs and the minimum number of reads required to pass filtering was set to 1 . After filtering, a total of 5347 ASVs were recovered. The sequences were filtered to remove non-bacterial, mitochondrial, and chloroplast sequences. Representative sequences were then assigned taxonomic classifications using a custom-trained V4-V5 16S rRNA naive Bayesian QIIME 2 classifier [26] trained on the 99\% Silva V132 database [27]. The MAFFT, mask, and FastTree protocols were used to generate phylogenetic trees of the aligned representative sequences in diversity analysis. The final feature table was rarefied to 1944 ASVs per sample. Three samples were discarded due to low read depth (NP at the 4 weeks' time point and replicates of NP during 8 weeks of sampling). Sample diversity metrics were generated for $\alpha$-diversity and $\beta$-diversity.

\subsection{Statistical Analysis}

The growth parameters of the plants $(n=12)$ with triplicates were subjected to a Student's $t$-test and one-way analysis of variance (ANOVA) using Duncan's post hoc test using SPSS version 26 . The same statistical analysis was performed using the gene counts 
obtained from PICRUSt 2. Taxonomic-based alpha diversity analysis was carried out using the 'microbiome' package to calculate community richness and evenness based on Shannon, Chao1, and richness diversity indices. Beta diversity analysis was performed based on the Bray-Curtis dissimilarity distance matrix along with non-metric multidimensional scaling (NMDS), which were subjected to Adonis having 999 permutations using the R package 'vegan' [28]. The above analysis results were illustrated on the plot utilizing the R package 'ggplot2' [29].

\section{Results}

\subsection{Phenotypic Effect of the AHL Soil Treatments on Ginseng Seedlings}

To observe the phenotypic effect of the AHL treatments, the growth parameters (shoot length, root length, shoot weight, root weight, and dry biomass) of the ginseng seedlings were recorded after two months of growth. The mortality rates of the baby ginseng plants after two months of development were also recorded. Surprisingly, no ginseng seedlings were found dead or exhibited disease in the mono-cropped ginseng soil (W), thus constituting a $0 \%$ mortality rate. Meanwhile, for the AHL treatments, $33.33 \%$ mortality rate was observed (4/12) in the C8-treated soil, while 8.33\% (1/12), and 0.25\% (3/12) mortality were observed in the C10- and C12-treated soils, respectively. However, the phenotypic result involving the shoot length, root length, wet, and dry biomass showed otherwise. Interestingly, the $\mathrm{W}$ sample showed the highest average root length $(14.67 \mathrm{~cm})$ but had the lowest average root weight $(0.51 \mathrm{~g})$. On the other hand, the treatment that involved C10 gave the highest average shoot length, shoot weight, and root weight $(6.06 \mathrm{~cm}, 0.61 \mathrm{~g}$, and $0.83 \mathrm{~g}$, respectively). For the dry biomass, the dry weight of both the roots and shoots was measured. Our results showed that treatment with $\mathrm{C} 10$ yielded the highest dry biomass ( $0.15 \mathrm{~g}$ of the shoot and $0.32 \mathrm{~g}$ of the root), which was statistically significant in comparison to $\mathrm{W}$, which showed the lowest value $(0.08 \mathrm{~g}$ of the dry shoot and $0.18 \mathrm{~g}$ of the dry root; $p<0.05)$. Overall, soils treated with AHL showed better plant growth, with $\mathrm{C} 10$ showing the best phenotypic results except for the root length. The results are summarized in Table 1 and Figure 1.

Table 1. Comparison of plant growth parameters using the root, shoot length, wet, and dry biomass contributed by $N$-acyl homoserine lactone treatments.

\begin{tabular}{ccccccc}
\hline Treatments & $\begin{array}{c}\text { Shoot Length } \\
(\mathbf{c m})\end{array}$ & $\begin{array}{c}\text { Root Length } \\
(\mathbf{c m})\end{array}$ & $\begin{array}{c}\text { Fresh Shoot } \\
\text { Biomass } \\
\text { (g/plant) }\end{array}$ & $\begin{array}{c}\text { Fresh Root } \\
\text { Biomass } \\
\text { (g/Plant) }\end{array}$ & $\begin{array}{c}\text { Dry Shoot } \\
\text { Biomass } \\
\text { (g/Plant) }\end{array}$ & $\begin{array}{c}\text { Dry Root } \\
\text { Biomass } \\
\text { (g/Plant) }\end{array}$ \\
\hline $\mathrm{W}$ & $4.87 \pm 0.14^{\mathrm{a}}$ & $14.67 \pm 0.95^{\mathrm{a}}$ & $0.45 \pm 0.09^{\mathrm{ab}}$ & $0.51 \pm 0.03^{\mathrm{a}}$ & $0.08 \pm 0.01^{\mathrm{a}}$ & $0.18 \pm 0.01^{\mathrm{a}}$ \\
$\mathrm{C} 8$ & $5.94 \pm 0.83^{\mathrm{a}}$ & $13.02 \pm 1.04^{\mathrm{a}}$ & $0.38 \pm 0.06^{\mathrm{a}}$ & $0.53 \pm 0.06^{\mathrm{a}}$ & $0.11 \pm 0.02^{\mathrm{ad}^{\mathrm{d}}}$ & $0.19 \pm 0.01^{\mathrm{a}}$ \\
$\mathrm{C} 10$ & $6.05 \pm 0.52^{\mathrm{a}}$ & $13.54 \pm 0.67^{\mathrm{a}}$ & $0.61 \pm 0.07^{\mathrm{b}}$ & $0.83 \pm 0.09^{\mathrm{c}}$ & $0.15 \pm 0.02^{\mathrm{d}}$ & $0.32^{\mathrm{a}} \pm 0.04^{\mathrm{c}}$ \\
$\mathrm{C} 12$ & $4.62 \pm 0.46^{\mathrm{a}}$ & $13.27 \pm 0.59^{\mathrm{a}}$ & $0.39 \pm 0.05^{\mathrm{a}}$ & $0.53 \pm 0.06^{\mathrm{a}}$ & $0.09 \pm 0.01^{\mathrm{a}}$ & $0.20 \pm 0.02^{\mathrm{a}}$ \\
\hline
\end{tabular}

Values are represented as the mean \pm standard error. Superscript letters indicate significant difference in one-way ANOVA and Duncan's multiple range test values $(p<0.05)$.

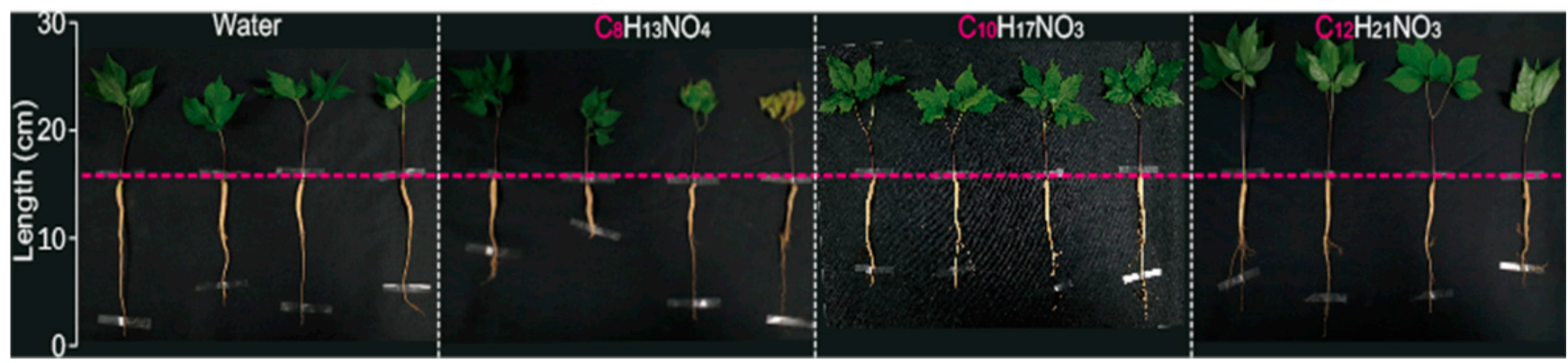

Figure 1. Effect of the treatment of the acyl-homoserine lactones $(\mathrm{C} 8, \mathrm{C} 10, \mathrm{C} 12)$ on the growth of ginseng. 


\subsection{Microbial Community Shifts Involving AHL Treatments on Ginseng Soil}

After processing the raw sequence files, we obtained a total of 273,217 reads, constituting 5347 ASVs. At the phylum level for the treated soil samples, we observed that Proteobacteria was the most dominant of the three treatments, C8 having $35.94 \%, \mathrm{C} 10$ $33.68 \%$, and $\mathrm{C} 12,33.76 \%$. Meanwhile, mono-cropped $\mathrm{W}$ showed a slightly lower percentage, although Proteobacteria also dominated it (30.87\%). Mono-cropped BS, on the other hand, was dominated by the Firmicutes phylum at $32.50 \%$. At the class level, all samples showed a high amount of $\gamma$-proteobacteria, with C 8 showing $28.58 \%$, C10 $23.58 \%$, and C12 $25.00 \%$, while the planted mono-cropped soil P showed $24.50 \%$, and mono-cropped BS showed $21.70 \%$. The family level was also checked, and similar results were obtained, with Pseudomonadaceae having the highest relative abundance for all samples $(15.53 \%, 10.12 \%$, $13.31 \%, 13.66 \%$, and $14.24 \%$ for C8, C10, C12, W, and BS samples, respectively) (Figure 2). Focusing on the top 10 taxa at the genus level, a high amount of Pseudomonas for all samples was observed. At the same time, we also see an increase of Catenibacterium and Bifidobacterium for W and BS samples. On the other hand, an increase in Pseudolabrys and Uncultured_Acidobacteriales was seen in the C10 and C12 samples.
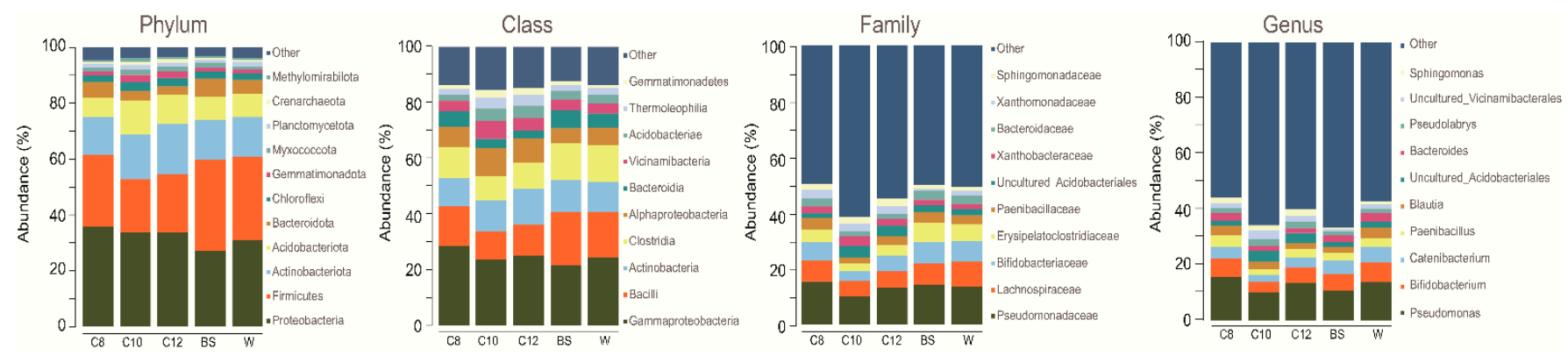

Figure 2. Relative abundance of different samples. A represents the relative abundance of the phylum, class, and family, and genus level.

The diversity of the groups was explained through different alpha diversity indices, such as Shannon, Chao1, and richness. All three alpha diversity indices showed a similar trend in the AHL-treated soils, exhibiting a lower diversity than BS and mono-cropped W samples. Although no statistically significant differences were found among the different treatments $(\mathrm{C} 8, \mathrm{C} 10$, and $\mathrm{C} 12)$, it is worth noting that the $\mathrm{C} 10$ sample, which gave the lowest diversity in the treated soils, was significantly different from $\mathrm{P}(p<0.05)$ (Figure 3). We also checked the alpha diversity at other time points $(2,4$, and 8 weeks). After two weeks of growth for all AHL-treated samples, we observed that the diversity was lower, while the opposite was seen in the W samples (Supplementary Figure S1). Similar trends were observed for both $\mathrm{C} 10$ and $\mathrm{C} 12$ samples, which increased in diversity after 4 and 8 weeks of growth. 
C8: N-Acyl homoserine lactone C8

C10: N-Acyl homoserine lactone $\mathrm{C} 10$

C12: N-Acyl homoserine lactone C12

BS: Bulk Soil

W : Water treated
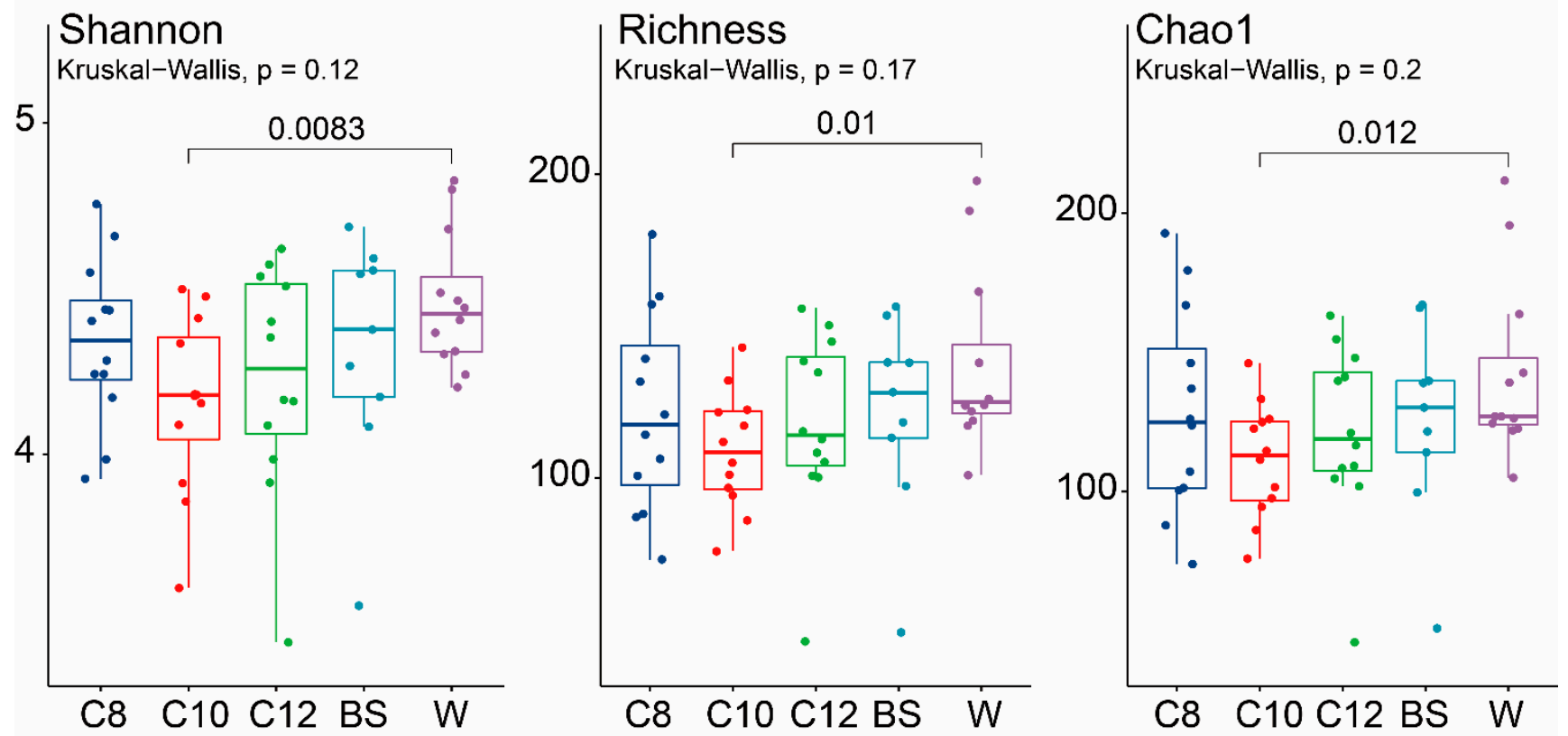

Figure 3. Total alpha diversity of soil microbial communities of the continuously mono-cropped treated with different moieties of acyl-homoserine lactones using Shannon, Richness, and Chao1 indices. The dark blue color shows the total alpha diversity for the C8 samples, while the red, green, light blue, and purple signify the C10, C12, BS, and W samples, respectively. The significance values were generated according to one-way ANOVA with Duncan's multiple range test values $(p<0.05)$.

The total variation in the diversity of the treatment groups is shown by the PCoA using Bray-Curtis dissimilarity and NMDS (stress $=0.19$ ) (Supplementary Figure S2). There was an inconsistent pattern observed during the initial time points. However, we observed a shift in the soil microbiome between BS-, $\mathrm{W}-$, and AHL-treated groups at different time points, especially after two weeks (Figure 4). The AHL-treated groups were scattered during different time points but were seemingly clustered with $\mathrm{W}$ after 4 and 8 weeks of growth. Analysis using Adonis showed that the treatments were scattered and significantly different after two weeks of growth $(p<0.05)$, while we found no significant difference after 4 and 8 weeks (Table 2). Although the scattering observed was significant only after two weeks, this result still supports our theory that the addition of AHL changed the microbiome of ginseng grown in a continuously cropped manner. 
C8: N-Acyl homoserine lactone C8

C10: N-Acyl homoserine lactone C10

C12: N-Acyl homoserine lactone C12

BS: Bulk Soil

W : Water treated
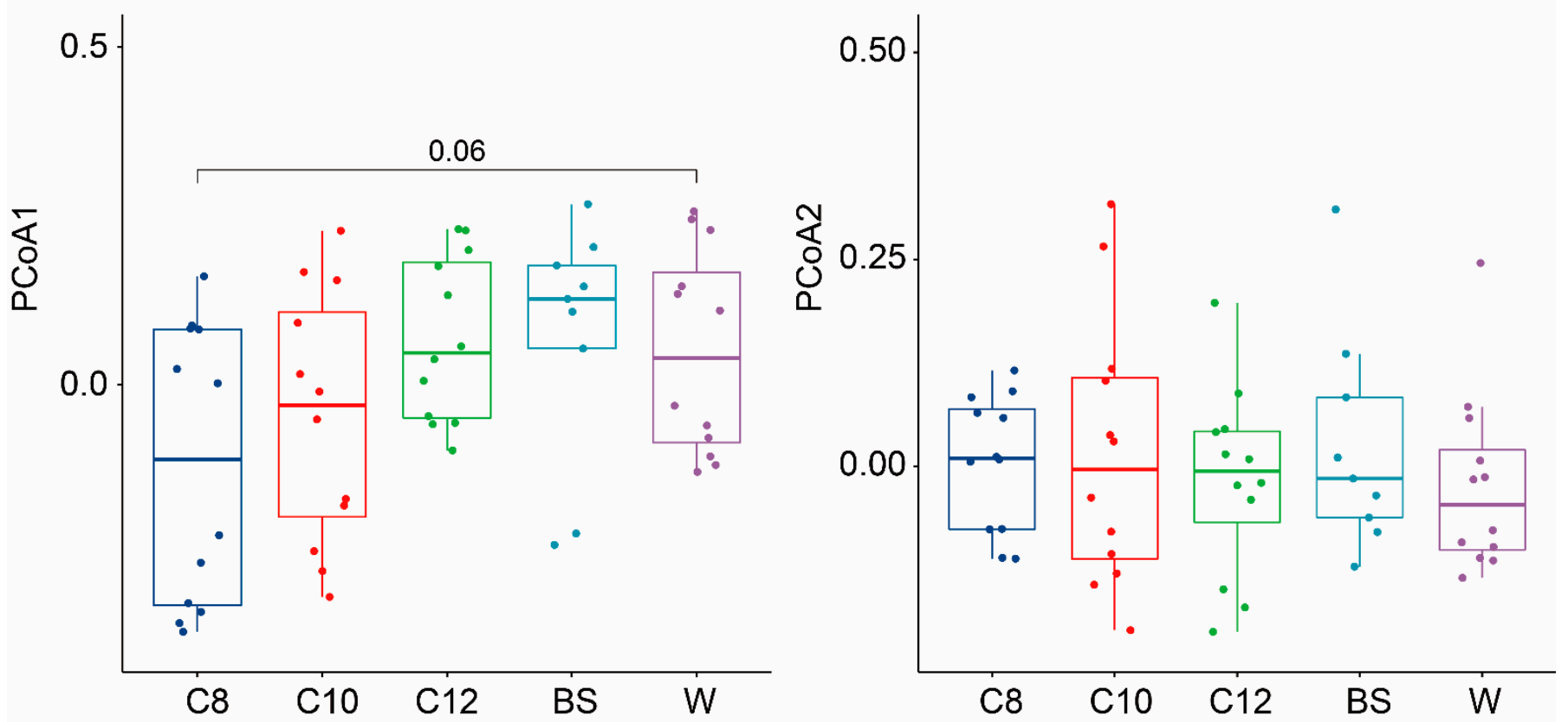

Figure 4. Boxplot of the Bray-Curtis dissimilarity for different samples across different sampling time points. The first plot shows the difference along the PCoA1 plane, while the second plot shows the difference along the PCoA2 plane. The dark blue color shows the total alpha diversity for the $\mathrm{C} 8$ samples, while the red, green, light blue, and purple colors signify the C10, C12, BS, and W samples, respectively. The significance values were generated according to one-way ANOVA with Duncan's multiple range test values $(p<0.05)$.

Table 2. PERMANOVA analysis using Adonis during the overall and different sampling points using Bray-Curtis dissimilarity distance values between $\mathrm{N}$-acyl homoserine lactone treatments $(\mathrm{C} 8, \mathrm{C} 10, \mathrm{C} 12)$ and the continuously mono-cropped ginseng soil $(\mathrm{W})$.

\begin{tabular}{|c|c|c|c|c|c|c|c|}
\hline & & DF & Sums of Sqs & Means Sqs & F. Model & $\mathbf{R}^{2}$ & $\operatorname{Pr}(>F)$ \\
\hline \multirow[t]{3}{*}{ Overall } & Treatment & 4 & 1.8139 & 0.45346 & 1.1343 & 0.08025 & 0.021 * \\
\hline & Residuals & 52 & 20.7888 & 0.39978 & & 0.91975 & \\
\hline & Total & 56 & 22.6026 & & & 1.00000 & \\
\hline \multirow[t]{3}{*}{2 weeks } & Treatment & 4 & 1.9865 & 0.49661 & 1.2304 & 0.32983 & $0.00 * *$ \\
\hline & Residuals & 10 & 4.0362 & 0.40362 & & 0.67017 & \\
\hline & Total & 14 & 6.0226 & & & 1.00000 & \\
\hline \multirow[t]{3}{*}{4 weeks } & Treatment & 4 & 1.2441 & 0.31103 & 0.98797 & 0.30512 & 0.539 \\
\hline & Residuals & 9 & 2.8334 & 0.31482 & & 0.69488 & \\
\hline & Total & 13 & 4.0775 & & & 1.00000 & \\
\hline \multirow[t]{3}{*}{8 weeks } & Treatment & 4 & 1.5079 & 0.37697 & 1.0388 & 0.34185 & 0.27 \\
\hline & Residuals & 8 & 2.9030 & 0.36288 & & 0.65815 & \\
\hline & Total & 12 & 4.4109 & & & 1.00000 & \\
\hline
\end{tabular}

Significance codes: $0.001^{\star * * \prime} 0.01^{\star * \prime}$ The significance values are based on 999 permutations. 


\subsection{Functional Gene Analysis Using PICRUSt2}

To better understand the shift in the microbiome, we analyzed the functional genes by employing the PICRUSt2. We used the Kyoto Encyclopedia of Genes and Genomes (KEGG) pathways to identify the functional genes present in our samples. We then focused on genes that are involved in QS, mainly those involving LuxI and LuxR. Afterward, the average gene counts per group were obtained. Surprisingly, the highest gene counts involved in the quorum-sensing pathway were found in the P samples significantly different from C10. There was an increase in the gene counts from the starting point until the fourth week of sampling for all samples except $\mathrm{C} 8$ and $\mathrm{W}$, while a sudden decrease of the quorum-sensing genes was observed in all samples for the last sampling date (week 8). The results are summarized in (Figure 5). We also analyzed which bacteria contributed on these genes, the top 10 genera were identified, and the data are summarized in Supplementary Table S1. We observed that the QS genes' top contributor was from the genus Pseudomonas for all the samples. A high amount of Escherichia-Shigella was observed in C10, while Variovorax was more predominant in samples $\mathrm{C} 8$ and $\mathrm{W}$.

A

Total predicted AHL genes in PICRUSt2

Total predicted AHL genes in PICRUSt2 during different sampling points

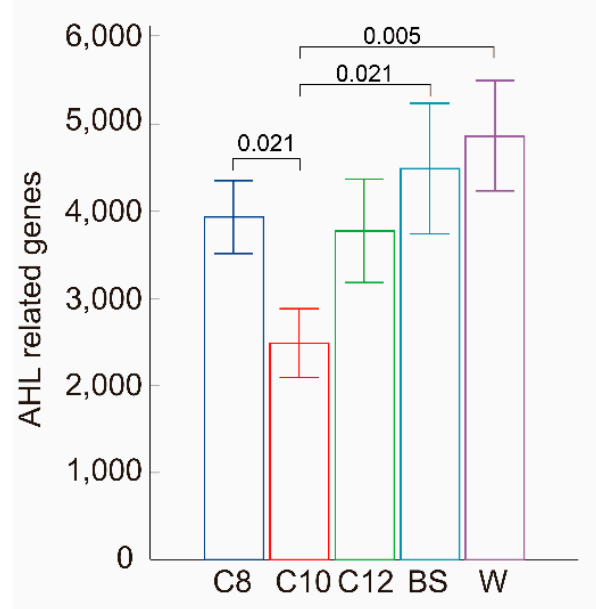

C8: N-Acyl homoserine lactone C8 BS: Bulk Soil

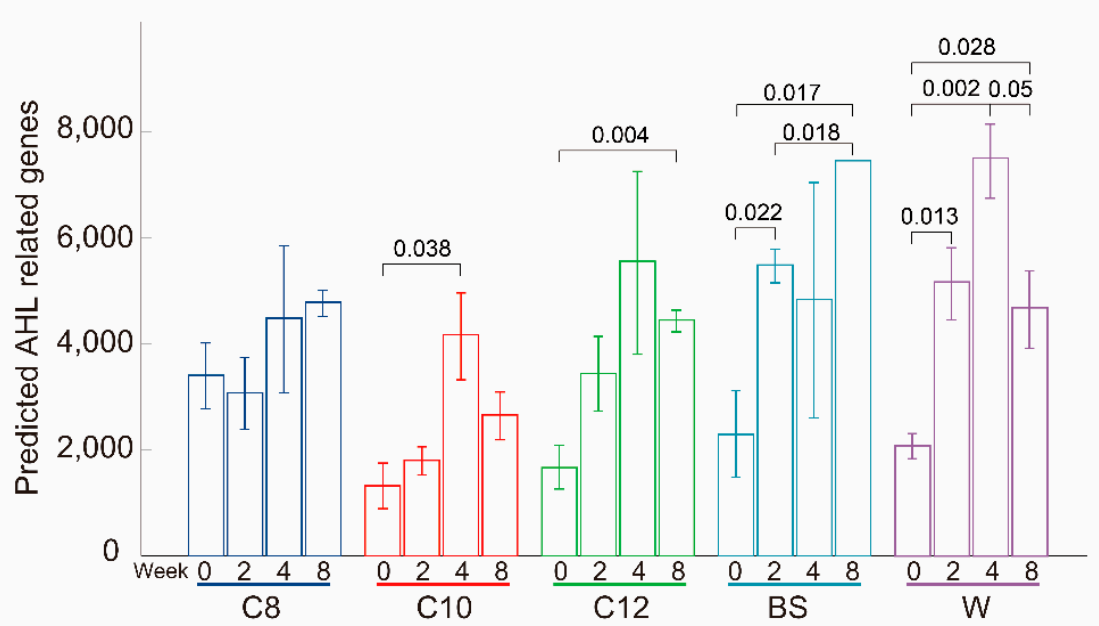

C10: N-Acyl homoserine lactone C10 $\square$ C12: N-Acyl homoserine lactone C12 W : Water treated

Figure 5. Bar graph showing the amount of quorum sensing related genes on the total (A) and different samples across different sampling time points (B). The significance values were generated according to one-way ANOVA with Duncan's multiple range test values $(p<0.05)$.

\section{Discussion}

A major challenge in the cultivation of ginseng plants is replanting, as it poses a considerable amount of loss in crops. This continuous monocropping is affected by the dynamic changes in soil microorganisms [30]. This study aimed to change the microbial community of soil used in monocropping through AHL treatment. A number of studies involving the addition of AHLs proved that it has positive effects on plant performance and beneficial plant responses [31]. The effect may vary depending on the length of side chains present in AHLs. Schenk and Schikora [32] showed that the use of AHLs having long side chains activated the oxylipin signaling pathway and priming for induced resistance. This was demonstrated by the involvement of defense hormones through AHL priming by inducing resistance in salicylic acid accumulation in tomatoes [33]. While Liu et al. [34] observed that AHLs with short side chains stimulate root length and antioxidative capacities in barley leaves [35]. A review done by Shrestha and Schikora [15] explains that there are 'AHL-primable' and 'AHL-non-primable' types depending on the response of plants 
through AHL priming. It has yet to be seen whether ginseng is a possible 'AHL-primable' crop, although our phenotypic results proved to be an excellent example of good ginseng growth when treated with AHLs, as can be determined by the data presented in Figure 1 and Table 1.

Observation of the microbiome composition of samples through $16 \mathrm{~S}$ rRNA amplicon sequencing revealed that at the class level, all samples showed a high amount of $\gamma$ proteobacteria. The treated samples were more enriched by $\gamma$-proteobacteria, which corroborates our treatment since the signaling molecules used were for AI-1 mediated signaling.

At the genus level, we found that Pseudomonas was dominant for all samples, although BS and W showed a higher amount of Catenibacterium and Bifidobacterium. Surprisingly, these genera, including Blautia, are more commonly found in the intestinal microbiomes of humans [36]. Although these genera are already present in soil [37], it is still unknown how they affect plant health. On the other hand, we observed an increase in Pseudolabrys which is included in the phylum proteobacteria [38] and Uncultured_Acidobacteriales in both C10 and C12 samples linked to promoting plant growth [39]. Moreover, a study by Cipriano et al. [40] and Chen et al. [41] showed that there was an increase in abundance of Pseudolabrys when a plant growth promoting bacteria was used for inoculation. It may be possible that, instead of plant growth promoting bacteria, the addition of AHLs helped in Pseudolabrys enrichment.

Meanwhile, analyzing the alpha diversity of the samples revealed a surprising result. Often, the health and quality of soil are linked with higher microbial diversity [42]. The diversity indices used in our study showed an overall decrease in the microbial diversity of AHL-treated groups compared to that of the continuously mono-cropped soil treated only with water (W), with C10 showing the lowest diversity. Studies have demonstrated a strong association between species richness, enhanced ecosystem functioning, and diversity of the soil microbiome [43]. Additionally, an increase in microbial diversity and richness showed a high correlation with plant productivity $[44,45]$ and plant resilience against soil-borne pathogen invasion $[46,47]$. It has also been suggested that higher bacterial diversity is correlated with higher functional diversity, which enables quick recovery during stress conditions and stimulates primary productivity [48]. However, we obtained the opposite result for ginseng, as shown in Figure 1. The overall diversity of the microbiome of plants with better growth was lower, as shown by the measured plant growth parameters (Table 1). The time series sampling showed that after two weeks of growth, the diversity in Shannon, Chao1, and Richness indices of the AHL-treated soils sharply decreased (Supplementary Figure S1). It is plausible that during the sampling periods at 4 and 8 weeks, the treatment effects wore off and were altered since the treatment was performed only during the initial period. Although it is noticeable that there was an increase in all the AHL treated soils in the last sampling date, while a decrease was observed in $\mathrm{W}$.

The NMDS plot showed that the initial microbiome of all samples was scattered at the start of the sampling point until two weeks of growth. Plant's 'collect and select' function for constructing a beneficial microbiome is mainly affected by the root exudates [10,49], which were affected by the AHL treatment in the second week of plant growth. The fourth and eighth weeks showed clustering of the W and BS samples, which suggests that their microbiomes were more similar. As demonstrated by the cluster of all the samples after 4 and 8 weeks of growth, there was no significant difference in their distances (Table 2), which indicated that the effect of treatment might have been lost. Thus, the customary microbiome for ginseng growth was attained.

Nonetheless, we observed changes in the soil microbiome that was treated with AHLs. According to the above studies [10,50], it is possible that crosstalk occurs between plants and AHL molecules, which may impact the root exudate, thus causing a significant effect on the ginseng rhizosphere microbiome. The combined effect of the root exudates of ginseng along with AHL treatment remains to be determined, although this finding could benefit the future of ginseng cultivation. 
The functional gene analysis through PICRUSt2 showed an elevated amount of quorum-sensing genes for the $\mathrm{W}$ samples. To determine which functional genes were present in the samples, we looked at the KEGG pathway involved in the AI-1 quorumsensing pathway and that involved in auxin synthesis. We obtained the list of genes involved and obtained the average gene count for each treatment. The higher counts were possibly associated with the higher diversity found in the $\mathrm{W}$ samples, since functional gene prediction is based on the ASVs. PICRUSt2 merges open-source tools to predict genomes of environmentally sampled $16 \mathrm{~S}$ rRNA gene sequences. The ASVs are then placed into a reference tree, which is used as the basis of functional predictions [49]. We understand that the functional genes found in the samples are based on predictions and only roughly represent what genes are present in the sample.

Moreover, studies using AHL for bacterial communities are difficult to conduct due to the complex interactions that occur within the community [51]. Analysis of the top 10 present genus, we observed a high abundance of Pseudolabrys and Unclassified_Acidobacteriales, Catenibacterium, Bifidobacterium, and Blautia. While there have been reports about the potential plant growth promoting properties of Pseudolabrys, we found no evidence of Unclassified_Acidobacteriales, Catenibacterium, Bifidobacterium, and Blautia with regard to plant growth promotion. We also analyzed the taxa that contributed to the QS genes based on PICRUSt2 and found Pseudomonas's most notable contributions for the samples. We also found a high amount of Sphingomonas in the C10 samples. Aside from exhibiting QS properties, Sphingomonas has also been reported to have plant growth promoting properties. A study by Kim et al. [52] showed that it exhibited plant growth promoting effect to rice. Another study done by Khan et al. [53] showed that the strain LK11 produced IAA, promoting tomato growth. It is possible that not only did they contribute to the QS gene abundance, but also contributed to the growth of the ginseng seedlings.

The results of the second week of sampling were still consistent with the increasing quorum-sensing gene counts for all samples, with the exception of $\mathrm{C} 8$ and lowering of the alpha diversity, while the beta diversity showed a scattered pattern, which suggests that the effect of the AHL treatment was still active during this time. The quorum-sensing genes had decreased by the subsequent sampling dates (8 weeks), at which time clustering was observed in the beta diversity (Figure 2). Although there was no mortality among the non-treated plant samples, a striking effect was observed on plant growth (shoot length, dry and wet biomass). A study done by Von Rad and colleagues [54] showed that treatment with oxo-C6-homoserine lactone provided the expression of genes associated with the auxin and cytokinin pathways. Moreover, a study conducted by Jung et al. [55] suggested that plant growth promotion affected the plant growth promotion trait of Serratia fonticola GS2, a quorum-sensing bacterial strain through transcriptome observation. In this study, the treated samples generally showed better growth in terms of the parameters used.

\section{Conclusions}

Our work focused on the shift of the soil microbiome involved in the ginseng soil rhizosphere. We tried to find dissimilarities between continuously mono-cropped ginseng soil and continuously mono-cropped AHL (C8, C10, and C12)-treated soils. Our phenotypic data of the ginseng plants showed better growth in the AHL-treated group, especially in C10. We also observed that in the AHL-treated group, the total alpha diversity was lower than in BS and W, which contrasts with the normal occurrence of a high microbial diversity known to be exhibited by healthy plants. The beta diversity also showed dispersion in the soil microbiome, especially after two weeks of treatment. The analysis of the quorum-sensing functional genes using PICRUST2 also showed an increased gene count in AHL-treated soils, albeit only at the second and fourth weeks. It is also possible that abiotic and biotic defense reactions were induced in ginseng seedlings via AHL treatment. However, further research is necessary for this. Overall, the results of this study shed light on the microbiome of continuously mono-cropped ginseng soil and the effect of three moieties of AHL. Although more studies are needed-including analyzing soil chemical 
properties and determining the root exudates of the ginseng plant-to determine whether AHL treatment can completely alleviate the replanting problem that arises in continuous cropping, this study suggests that the use of AHLs can shift the soil microbiome and shows promise in supporting the growth of ginseng.

Supplementary Materials: The following are available online at https:/ / www.mdpi.com/article/ 10.3390 /agronomy11112177/s1: Supplementary Table S1. List of top 10 genus that contributed to QS functional genes using PICRUSt2 Figure S1: Alpha diversity of the different samples across the other sampling times $(0,2,4$, and 8 weeks) using Richness, Chao1, and Shannon indices. The dark blue color shows the total alpha diversity for the $\mathrm{C} 8$ samples, while the red, green, light blue, and purple colors signify C10, C12, BS, and W samples, respectively.; Figure S2: NMDS visualizations of beta diversity analysis using the Bray-Curtis metric. The ellipses represent the different samples across sampling dates. The different colors represent the different weeks of sampling orange for the initial sampling, green for two weeks after growth, blue and purple for the fourth and eighth weeks of sampling, respectively.

Author Contributions: Conceptualization, J.-H.S., G.-S.P., and B.-K.J.; Methodology, J.-C.I., M.-K.P., and Y.-J.P.; Software, M.-S.K. and G.-U.K.; Validation, J.-C.I. and M.-K.P.; Formal analysis, J.-C.I., M.-K.P., and M.-S.K.; Investigation, J.-C.I. and M.-K.P.; Resources, Y.-J.P. and G.-U.K.; Data curation, J.-C.I. and T.-H.P.; Writing-Original draft preparation, J.-C.I. and M.-K.P.; Writing-Review and editing, J.-H.S.; Visualization, M.-K.P. and M.-S.K.; Supervision, J.-H.S.; Project administration, J.-H.S.; Funding acquisition, J.-H.S. All authors have read and agreed to the published version of the manuscript.

Funding: This work was supported by the regional researcher program through the National Research Foundation (NRF), funded by the Ministry of Education (NRF-2020R1I1A307452212), the Commercialization Promotion Agency for R\&D Outcomes (COMPA) grant funded by the Ministry of Science and ICT (R\&D project no. 1711139487), the project to train professional personnel in biological materials by the Ministry of Environment, and the Korea Institute of Planning and Evaluation for Technology in Food, Agriculture and Forestry (IPET) through Crop Viruses and Pests Response Industry Technology Development Program, funded by Ministry of Agriculture, Food and Rural Affairs (MAFRA) (321097-3).

Data Availability Statement: All raw sequencing data used in this study were uploaded in the NCBI Sequence Read Archive (SRA) under the accession number PRJNA761853.

Acknowledgments: We express our appreciation and especially thank the support and cooperation of the KNU NGS core facility regarding microbiome sequencing.

Conflicts of Interest: The authors declare no conflict of interest.

\section{References}

1. Wen, J.; Zimmer, E.A. Phylogeny and biogeography of Panax L. (the ginseng genus, Araliaceae): Inferences from ITS sequences of nuclear ribosomal DNA. Mol. Phylogenet. Evol. 1996, 6, 167-177. [CrossRef] [PubMed]

2. Yun, T.K. Brief introduction of Panax ginseng CA Meyer. J. Korean Med. Sci. 2001, 16, S3-S5. [CrossRef] [PubMed]

3. Kim, J.H. Cardiovascular diseases and Panax ginseng: A review on molecular mechanisms and medical applications. J. Ginseng Res. 2012, 36, 16. [CrossRef] [PubMed]

4. Ma, H.; Liu, D.; Sun, H. Deciphering microbiome related to rusty roots of Panax ginseng and evaluation of antagonists against pathogenic Ilyonectria. Front. Microbiol. 2019, 10, 1350.

5. Wei, X.; Wang, X.; Cao, P.; Gao, Z.; Chen, A.J.; Han, J. Microbial community changes in the rhizosphere soil of healthy and rusty Panax ginseng and discovery of pivotal fungal genera associated with rusty roots. Biomed Res. Int. 2020. [CrossRef]

6. Atkinson, D.; Watson, C.A. The beneficial rhizosphere: A dynamic entity. Appl. Soil Ecol. 2000, 15, 99-104. [CrossRef]

7. Whipps, J.M. Microbial interactions and biocontrol in the rhizosphere. J. Exp. Bot. 2001, 52 (Suppl. 1), 487-511. [CrossRef]

8. Song, C.; Zhu, F.; Carrión, V.J.; Cordovez, V. Beyond plant microbiome Composition: Exploiting microbial functions and plant traits via integrated approaches. Front. Bioeng. Biotechnol. 2020, 8. [CrossRef]

9. Dong, L.L.; Niu, W.H.; Wang, R.; Xu, J.; Zhang, L.J.; Zhang, J.; Chen, S.L. Changes of diversity and composition of fungal communities in rhizosphere of Panax ginseng. Zhongguo Zhong Yao Za Zhi 2017, 42, 443-449. [CrossRef]

10. Korenblum, E.; Dong, Y.; Szymanski, J.; Panda, S.; Jozwiak, A.; Massalha, H.; Meir, S.; Rogachev, I.; Aharoni, A. Rhizosphere microbiome mediates systemic root metabolite exudation by root-to-root signaling. Proc. Natl. Acad. Sci. USA 2020, 117, 3874-3883. [CrossRef] 
11. Raaijmakers, J.M.; Paulitz, T.C.; Steinberg, C.; Alabouvette, C.; Moënne-Loccoz, Y. The rhizosphere: A playground and battlefield for soilborne pathogens and beneficial microorganisms. Plant Soil. 2009, 321, 341-361. [CrossRef]

12. Lareen, A.; Burton, F.; Schäfer, P. Plant root-microbe communication in shaping root microbiomes. Plant Mol. Biol. 2016, 90, 575-587. [CrossRef]

13. Antoun, H.; Kloepper, J.W. Encyclopedia of Genetics; Academic: New York, NY, USA, 2001.

14. Mohanram, S.; Kumar, P. Rhizosphere microbiome: Revisiting the synergy of plant-microbe interactions. Ann. Clin. Microbiol. 2019, 69, 307-320. [CrossRef]

15. Shrestha, A.; Schikora, A. AHL-priming for enhanced resistance as a tool in sustainable agriculture. FEMS Microbiol. Ecol. 2020, 96, fiaa226. [CrossRef]

16. Ng, W.L.; Bassler, B.L. Bacterial quorum-sensing network architectures. Annu. Rev. Genet. 2009, 43, 197-222. [CrossRef]

17. Lazazzera, B.A.; Grossman, A.D. The ins and outs of peptide signaling. Trends Microbiol. 1998, 6, 288-294. [CrossRef]

18. Von Bodman, S.B.; Bauer, W.D.; Coplin, D.L. Quorum sensing in plant-pathogenic bacteria. Annu. Rev. Phytopathol. 2003, 41, 455-482. [CrossRef] [PubMed]

19. Papenfort, K.; Bassler, B.L. Quorum sensing signal-response systems in Gram-negative bacteria. Nat. Rev. Microbiol. 2016, 14, 576. [CrossRef] [PubMed]

20. Zarkani, A.A.; Stein, E.; Röhrich, C.R.; Schikora, M.; Evguenieva-Hackenberg, E.; Degenkolb, T.; Vilcinskas, A.; Klug, G.; Kogel, K.-H.; Schikora, A. Homoserine lactones influence the reaction of plants to rhizobia. Int. J. Mol. Sci. 2013, 14, 17122-17146. [CrossRef] [PubMed]

21. Shrestha, A.; Elhady, A.; Adss, S.; Wehner, G.; Böttcher, C.; Heuer, H.; Ordon, F.; Schikora, A. Genetic differences in barley govern the responsiveness to N-Acyl homoserine lactone. Phytobiomes J. 2019, 3, 191-202. [CrossRef]

22. Shrestha, A.; Grimm, M.; Ojiro, I.; Krumwiede, J.; Schikora, A. Impact of quorum sensing molecules on plant growth and immune system. Front. Microbiol. 2020, 11, 1545. [CrossRef]

23. Liang, X.; Wagner, R.E.; Li, B.; Zhang, N.; Radosevich, M. Quorum sensing signals alter in vitro soil virus abundance and bacterial community composition. Front. Microbiol. 2020, 11, 1287. [CrossRef] [PubMed]

24. Bolyen, E.; Rideout, J.R.; Dillon, M.R.; Bokulich, N.A.; Abnet, C.C.; Al-Ghalith, G.A.; Bai, Y. Reproducible, interactive, scalable and extensible microbiome data science using QIIME 2. Nat. Biotechnol. 2019, 37, 852-857. [CrossRef]

25. Amir, A.; McDonald, D.; Navas-Molina, J.A.; Kopylova, E.; Morton, J.T.; Zech Xu, Z.; Kightley, E.P.; Thompson, L.R.; Hyde, E.R.; Gonzalez, A.; et al. Deblur rapidly resolves single-nucleotide community sequence patterns. MSystems 2017, 2, e00191-16. [CrossRef] [PubMed]

26. Bokulich, N.A.; Kaehler, B.D.; Rideout, J.R.; Dillon, M.; Bolyen, E.; Knight, R.; Huttley, G.A.; Gregory Caporaso, J. Optimizing taxonomic classification of marker-gene amplicon sequences with QIIME 2's q2-feature-classifier plugin. Microbiome 2018, 6, 90. [CrossRef]

27. Pruesse, E.; Quast, C.; Knittel, K.; Fuchs, B.M.; Ludwig, W.; Peplies, J.; Glöckner, F.O. SILVA: A comprehensive online resource for quality checked and aligned ribosomal RNA sequence data compatible with ARB. Nucleic Acids Res. 2007, 35, 7188-7196. [CrossRef]

28. Dixon, P. VEGAN, a package of R functions for community ecology. Appl. Veg. Sci. 2003, 14, 927-930. [CrossRef]

29. Wickham, H. ggplot2. Comput. Stat. Data Anal. 2011, 3, 180-185.

30. Lei, H.; Liu, A.; Hou, Q.; Zhao, Q.; Guo, J.; Wang, Z. Diversity patterns of soil microbial communities in the Sophora flavescens rhizosphere in response to continuous monocropping. BMC Microbiol. 2020, 20, 272. [CrossRef]

31. Hartmann, A. Quorum sensing N-acyl-homoserine lactone signal molecules of plant beneficial Gram-negative rhizobacteria support plant growth and resistance to pathogens. Rhizosphere 2020, 16, 100258. [CrossRef]

32. Schenk, S.T.; Schikora, A. AHL-priming functions via oxylipin and salicylic acid. Front. Plant Sci. 2015, 5, 784. [CrossRef] [PubMed]

33. Hartmann, A.; Rothballer, M.; Hense, B.A.; Schröder, P. Bacterial quorum sensing compounds are important modulators of microbe-plant interactions. Front. Plant Sci. 2014, 5, 131. [CrossRef]

34. Liu, F.; Bian, Z.; Jia, Z.; Zhao, Q.; Song, S. The GCR1 and GPA1 participate in promotion of Arabidopsis primary root elongation induced by $\mathrm{N}$-acyl-homoserine lactones, the bacterial quorum-sensing signals. Mol. Plant-Microbe Interact. 2012, 25, 677-683. [CrossRef]

35. Götz-Rösch, C.; Sieper, T.; Fekete, A.; Schmitt-Kopplin, P.; Hartmann, A.; Schröder, P. Influence of bacterial N-acyl-homoserine lactones on growth parameters, pigments, antioxidative capacities and the xenobiotic phase II detoxification enzymes in barley and yam bean. Front. Plant Sci. 2015, 6, 205. [CrossRef]

36. Yang, J.; Martínez, I.; Walter, J.; Keshavarzian, A.; Rose, D.J. In vitro characterization of the impact of selected dietary fibers on fecal microbiota composition and short chain fatty acid production. Anaerobe 2013, 23, 74-81. [CrossRef] [PubMed]

37. Maquia, I.S.; Fareleira, P.; Videira E Castro, I.; Brito, D.; Soares, R.; Chaúque, A.; Ferreira-Pinto, M.M.; Lumini, E.; Berruti, A.; Ribeiro, N.S.; et al. Mining the Microbiome of Key Species from African Savanna Woodlands: Potential for Soil Health Improvement and Plant Growth Promotion. Microorganisms 2020, 8, 1291. [CrossRef] [PubMed]

38. Kämpfer, P.; Young, C.C.; Arun, A.B.; Shen, F.T.; Jäckel, U.; Rossello-Mora, R.; Rekha, P.D. Pseudolabrys taiwanensis gen. nov., sp. nov., an alphaproteobacterium isolated from soil. Int. J. Syst. Evol. Microbiol. 2006, 56, 2469-2472. [CrossRef] [PubMed] 
39. Song, Q.; Song, X.; Deng, X.; Luo, J.; Wang, J.; Min, K.; Song, R. Effects of Plant Growth Promoting Rhizobacteria Microbial Inoculants on the Growth, Rhizosphere Soil Properties, and Bacterial Community of Pinus sylvestris var. mongolica Annual Seedlings. Res. Sq. 2020. [CrossRef]

40. Cipriano, M.A.; Lupatini, M.; Lopes-Santos, L.; da Silva, M.J.; Roesch, L.F.; Destéfano, S.A.; Freitas, S.S.; Kuramae, E.E. Lettuce and rhizosphere microbiome responses to growth promoting Pseudomonas species under field conditions. FEMS Microbiol. Ecol. 2016, 92, fiw197. [CrossRef]

41. Chen, L.; Hao, Z.; Li, K.; Sha, Y.; Wang, E.; Sui, X.; Mi, G.; Tian, C.; Chen, W. Effectsof growth-promoting rhizobacteria on maize growth and rhizosphere microbial community under conservation tillage in Northeast China. Microbial Biotechnol. 2021, 14, 535-550. [CrossRef] [PubMed]

42. Johnson, J.; Rychahou, P.; Sviripa, V.M.; Weiss, H.L.; Liu, C.; Watt, D.S.; Evers, B.M. Characterizing changes in soil microbiome abundance and diversity due to different cover crop techniques. PLoS ONE 2020, 15, e0232453.

43. Wagg, C.; Schlaeppi, K.; Banerjee, S.; Kuramae, E.E.; van der Heijden, M.G. Fungal-bacterial diversity and microbiome complexity predict ecosystem functioning. Nat. Commun. 2019, 10, 4841. [CrossRef] [PubMed]

44. Lau, J.A.; Lennon, J.T. Evolutionary ecology of plant-microbe interactions: Soil microbial structure alters selection on plant traits. New Phytol. 2011, 192, 215-224. [CrossRef] [PubMed]

45. van der Heijden, M.G.; Wagg, C. Soil microbial diversity and agro-ecosystem functioning. Plant Soil. 2013, 363, 1-5. [CrossRef]

46. Jousset, A.; Schmid, B.; Scheu, S.; Eisenhauer, N. Genotypic richness and dissimilarity opposingly affect ecosystem functioning. Ecol. Lett. 2011, 14, 537-545. [CrossRef] [PubMed]

47. Mendes, R.; Kruijt, M.; De Bruijn, I.; Dekkers, E.; van der Voort, M.; Schneider, J.H.; Raaijmakers, J.M. Deciphering the rhizosphere microbiome for disease-suppressive bacteria. Science 2011, 332, 1097-1100. [CrossRef]

48. Fierer, N.; Ladau, J.; Clemente, J.C.; Leff, J.W.; Owens, S.M.; Pollard, K.S.; McCulley, R.L. Reconstructing the microbial diversity and function of pre-agricultural tallgrass prairie soils in the United States. Science 2013, 342, 621-624. [CrossRef]

49. Douglas, G.M.; Maffei, V.J.; Zaneveld, J.R.; Yurgel, S.N.; Brown, J.R.; Taylor, C.M.; Huttenhower, C.; Langille, M. PICRUSt2 for prediction of metagenome functions. Nat. Biotechnol. 2020, 38, 685-688. [CrossRef]

50. Zhalnina, K.; Louie, K.B.; Hao, Z.; Mansoori, N.; da Rocha, U.N.; Shi, S.; Brodie, E.L. Dynamic root exudate chemistry and microbial substrate preferences drive patterns in rhizosphere microbial community assembly. Nat. Microbiol. 2018, 3, 470-480. [CrossRef]

51. Abisado, R.G.; Benomar, S.; Klaus, J.R.; Dandekar, A.A.; Chandler, J.R. Bacterial quorum sensing and microbial community interactions. MBio 2018, 9, e02331-17. [CrossRef]

52. Kim, Y.J.; Lim, J.; Sukweenadhi, J.; Seok, J.W.; Lee, S.W.; Park, J.C.; Taizhanova, A.; Kim, D.; Yang, D.C. Genomic characterization of a newly isolated rhizobacteria Sphingomonas panacis reveals plant growth promoting effect to rice. Biotechnol. Bioprocess Eng. 2019, 24, 119-125. [CrossRef]

53. Khan, A.L.; Waqas, M.; Kang, S.M.; Al-Harrasi, A.; Hussain, J.; Al-Rawahi, A.; Al-Khiziri, S.; Ullah, I.; Ali, L.; Jung, H.Y.; et al. Bacterial endophyte Sphingomonas sp. LK11 produces gibberellins and IAA and promotes tomato plant growth. J. Microbiol. 2014, 52, 689-695. [CrossRef]

54. Von Rad, U.; Klein, I.; Dobrev, P.I.; Kottova, J.; Zazimalova, E.; Fekete, A.; Hartmann, A.; Schmitt-Kopplin, P.; Durner, J. Response of Arabidopsis thaliana to N-hexanoyl-DL-homoserine-lactone, a bacterial quorum sensing molecule produced in the rhizosphere. Planta 2008, 229, 73-85. [CrossRef] [PubMed]

55. Jung, B.K.; Ibal, J.C.; Pham, H.Q.; Kim, M.C.; Park, G.S.; Hong, S.J.; Jo, H.W.; Park, C.E.; Choi, S.D.; Jung, Y.; et al. Quorum sensing system affects the plant growth promotion traits of serratia fonticola GS2. Front. Microbiol. 2020, 11, 536865. [CrossRef] [PubMed] 